\title{
Spectrophotometric determination of platelet counts in platelet-rich plasma
}

\author{
Yutaka Kitamura', Masashi Suzuki', Tsuneyuki Tsukioka' ${ }^{1}$ Kazushige Isobe ${ }^{1}$, Tetsuhiro Tsujino' ${ }^{1}$ Taisuke Watanabe', \\ Takao Watanabe ${ }^{1}$, Hajime Okudera ${ }^{1}$, Koh Nakata ${ }^{2}$, Takaaki Tanaka ${ }^{3}$ and Tomoyuki Kawase ${ }^{4^{*}}$ (D)
}

\begin{abstract}
Background: Platelet-rich plasma (PRP) is widely used in regenerative dentistry and other medical fields. However, its effectiveness has often been questioned. For better evaluation, the quality of individual PRP preparations should be assured prior to use. We proposed a spectrophotometric method for determination of platelet counts and validated its applicability using two types of PRP preparations.

Methods: Blood samples were obtained from healthy male volunteers and pure PRP (P-PRP) and leukocytes-rich PRP (L-PRP) were prepared using the double-spin method. In serial dilutions, platelet counts in P-PRP and L-PRP were determined using an automated hematology analyzer and a compact spectrophotometer. For validation, PPRP and L-PRP independently prepared by three well-trained operators were used for comparison of the calculated and measured platelet counts.

Results: In the two types of PRP samples evaluated, platelet counts were almost equal and greater amount of both white blood cells (WBCs) and red blood cells (RBCs) were included in L-PRP preparations. The calibration curve obtained from serially diluted P-PRP showed a strong correlation $\left(R^{2}=0.995\right)$, whereas that of L-PRP was relatively weaker $\left(R^{2}=0.975\right)$. In validation testing, the scatter plot of the calculated platelet counts versus the measured values showed a strong correlation in P-PRP $\left(R^{2}=0.671\right)$, whereas that of L-PRP showed a much weaker correlation $\left(R^{2}=0.0605\right)$.
\end{abstract}

Conclusions: This method can precisely determine platelet counts in PRP preparations when the inclusion of WBCs or RBCs is minimized. Therefore, we recommend that clinicians use this method for quality assurance of individual PRP preparations.

Keywords: Platelet, Count, Spectrophotometry, Leukocytes, Red blood cells, Quality assurance

\section{Background}

Almost two decades have passed since platelet concentrates, such as platelet-rich plasma (PRP), were first introduced to the field of regenerative medicine by Marx et al. [1]. To date, PRP has been modified to create different variations and has increasingly been used in various fields of regenerative therapy around the world. However, negative data obtained from clinical applications of PRP have often been reported, leading to controversy regarding the predictability of PRP therapy [2].

\footnotetext{
* Correspondence: kawase@dent.niigata-u.ac.jp

${ }^{4}$ Division of Oral Bioengineering, Institute of Medicine and Dentistry, Niigata

University, Niigata, Japan

Full list of author information is available at the end of the article
}

Especially in cases of skeletal regeneration, the efficacy of PRP has been controversial [3-9].

One possible major reason behind this debate is the lack of large controlled clinical trials [2] or randomized clinical trials. Because there is no consensus regarding the indications and contraindications for PRP therapy, it is theoretically difficult to design appropriate experiments. In addition, there are no generally accepted guidelines on how to evaluate the condition of application sites. The second major reason, which has frequently been used as a possible explanation (actually, an "excuse") for unexpected clinical results in many clinical case reports, is individual difference. This is highly conceivable, but not convincingly supported by scientific evidence in individual cases. The third major reason is 
the lack of consensus regarding PRP preparation protocols [2]. Recent advances in the development of various automated preparation devices and kits are expected to reduce not only the labor of the operator but also technique-dependent variation of PRP quality. However, it should be noted that these devices cannot standardize PRP quality. In other words, it is not guaranteed that the quality of individual PRP preparations depends specifically on individual preparation devices. In fact, it is well-known that PRP and its derivatives prepared using the same devices do not necessarily induce similar clinical results.

In Japan, a new regulatory framework for PRP therapy was established in 2014. However, no evaluation indexes for PRP quality, except for aseptic handling to ensure sterility, are indicated in the regulations. In our recent review article [10], we highlighted the necessity of PRP quality indexes. The primary index is platelet counts. Specifically, it is best to check platelet counts prior to use. To assess PRP quality in clotted PRP derivatives, such as platelet-rich fibrin (PRF), we recently developed a direct counting method for platelets contained in fibrin clots [11]. However, only a few clinicians possess automated hematology analyzers (AHAs) or similar electronic devices that can be used to determine platelet counts accurately without bias or technical error.

In this study, we focused on the possibility of spectrophotometric determination and validated the applicability of the proposed method on platelet counts in PRP preparations. This idea was based on bacterial cell counting [12] and a similar challenge was reported in 1992 [13]. However, this optical method has not been further modified for PRP as a grafting material for regenerative therapy in accordance with the policy of quality assurance. Based on the count of white blood cells (WBCs) and red blood cells (RBCs) included in PRP preparations, we categorized PRP preparations into two types as follows: pure PRP (P-PRP) and leukocyte-rich PRP (L-PRP) [14-16]. As not only platelets but also WBCs are concentrated in L-PRP, we hypothesized that the inclusion of WBCs at higher levels could markedly interfere with this spectrophotometric determination. As predicted, we validated the applicability of our proposed method by precisely determining platelet counts in P-PRP, but not L-PRP.

\section{Methods}

\section{Preparation of P-PRP and L-PRP}

Blood samples were collected from 11 non-smoking healthy male volunteers aged 33 to 69 years. The study design and consent forms for all the procedures were approved by the ethics committee for human participants at the Niigata University School of Medicine (Niigata, Japan) in accordance with the Helsinki Declaration of 1964 as revised in 2013.
Peripheral blood $(\sim 9 \mathrm{~mL})$ was collected into plastic vacuum plain blood collection tubes (Neotube; NIPRO, Osaka, Japan) containing $1 \mathrm{~mL}$ of the A-formulation of acid-citrate-dextrose (ACD-A; Terumo, Tokyo, Japan). The whole-blood samples were stored using a rotating agitator at ambient temperature and were used within $36 \mathrm{~h}$. The whole-blood samples were centrifuged at $533 \times g$ for $10 \mathrm{~min}$ (first low-speed spin). For P-PRP preparation, the upper plasma fraction, which was approximately $2 \mathrm{~mm}$ beyond the interface between the plasma and $\mathrm{RBC}$ fractions, was transferred into $2-\mathrm{mL}$ sample tubes for the second high-speed spin $(2656 \times g, 5 \mathrm{~min})$. For L-PRP preparation, the upper plasma fraction was transferred along with a buffy coat and the surface of the $\mathrm{RBC}$ fraction for the second spin. Prior to the second spin, $0.5 \mu \mathrm{g} / \mathrm{mL}$ prostaglandin $\mathrm{E}_{1}\left(\mathrm{PGE}_{1}\right.$ ) (Wako Pure Chemicals, Osaka, Japan) was added to each sample to prevent platelet aggregation. After centrifugation, 50$70 \%$ of the supernatant (PPP) was removed, and platelets (and other blood cells, if any) were resuspended in the remaining PPP fraction.

The numbers of platelets and other blood cells in the whole-blood samples and PRP preparations were determined using an AHA (pocH 100iV, Sysmex, Kobe, Japan).

\section{Spectrophotometric determination of platelet counts and calibration curves}

P-PRP and L-PRP preparations were serially diluted with the corresponding amount of PPP. The series of P-PRP and L-PRP dilutions were first subjected to measurement using the AHA and subsequently subjected to measurement with a compact scanning probe microscope (SPM; PiCOSCOPE, Ushio Inc., Tokyo, Japan) (Fig. 1). The SPM can be operated by remote control through a specific application installed on smart devices,

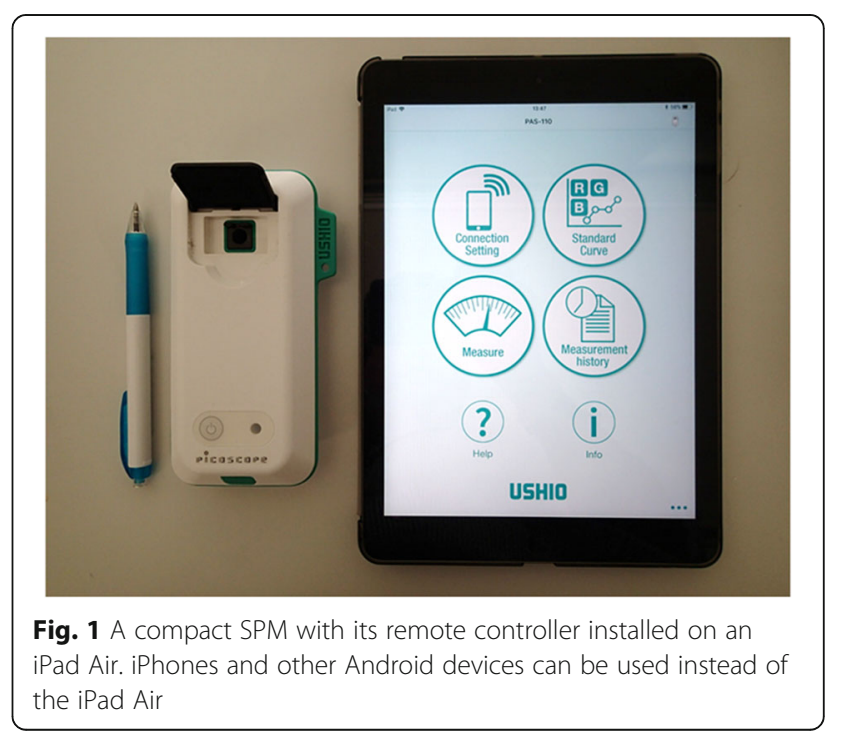




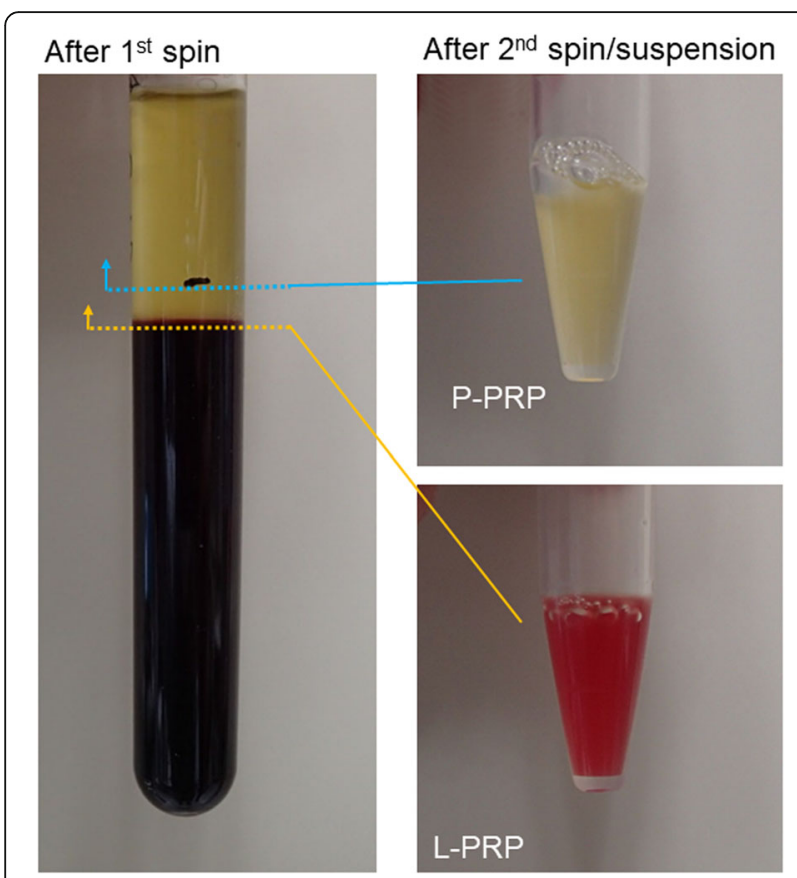

Fig. 2 The appearance of blood sampled after gravity fractionation and the resulting P-PRP and L-PRP. In the first low-speed spin, samples were centrifuged for $10 \mathrm{~min}$ at $533 \times \mathrm{g}$. For P-PRP preparation, the upper plasma fraction, which was $2 \mathrm{~mm}$ beyond the interface between plasma and RBC fractions, was transferred into sample tubes for the second high-speed spin (2656xg, 5 min). In contrast, for L-PRP preparation, the upper plasma fraction including the buffy coat and the surface of the RBC fraction was used for the second spin. The supernatant (PPP) was excluded by $50-70 \%$, and platelets were resuspended in the remaining PPP fraction including the iPad Air (Apple, Cupertino, CA, USA). PRP samples were transferred into $0.2 \mathrm{~mL}$ highly transparent PCR tubes (Nippon Genetics Co., Ltd., Tokyo, Japan) and were measured at $615 \mathrm{~nm}$ (range of wavelength 570-660 nm).

Using the data obtained with both the AHA and SPM, scattered plots were created to examine correlations and obtain formulas to calculate platelet counts.

\section{Validation testing}

P-PRP and L-PRP preparations were independently prepared from the 11 donors by three well-trained operators. Platelet counts were first determined using the AHA and aliquots of the PRP preparations were measured using the SPM. Platelet counts were calculated with the appropriate formulas and were compared with the measured platelet counts.

\section{Statistical analysis}

The data are expressed as mean \pm standard deviation (SD). For two-group comparisons, statistical analyses were conducted to compare the mean values using the Student's $t$ test (SigmaPlot 12.5; Systat Software, Inc., San Jose, CA, USA). $P$ values of $<0.05$ were considered statistically significant. The strength of a linear association between measured platelet counts and absorbance values was evaluated using the Pearson correlation coefficient $(R)$. Based on these data, we obtained formulas for calculating platelet counts using absorbance values. Additionally, possible correlations between platelets and RBCs or WBCs and those between measured and calculated platelet counts were also evaluated using the Pearson correlation coefficient.
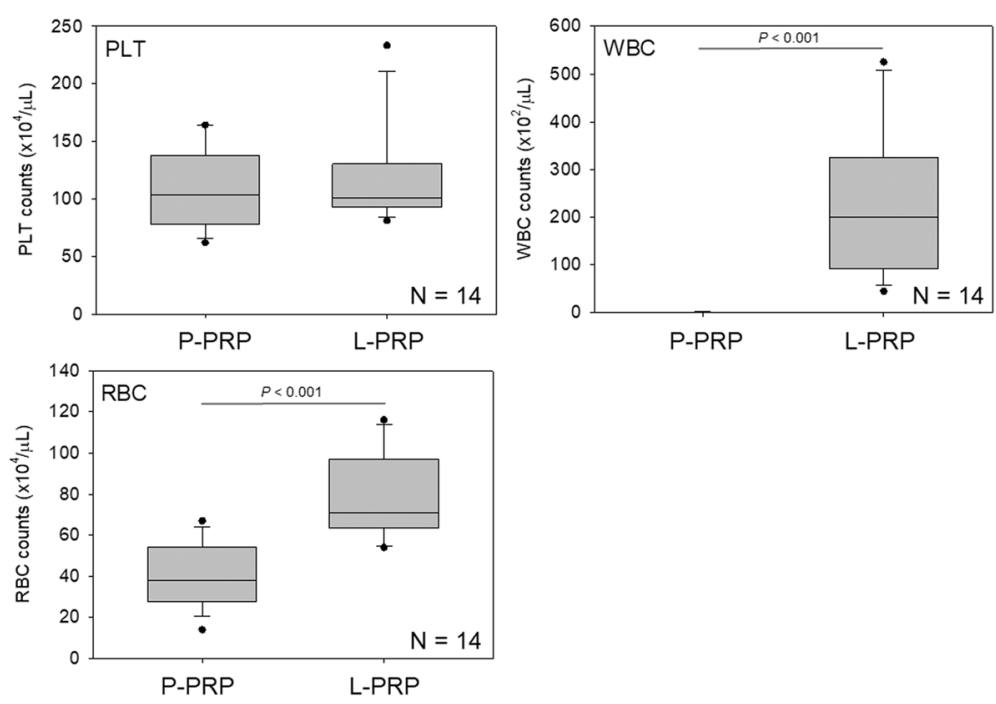

Fig. 3 Counts of platelets (PLT), WBCs, and RBCs in P-PRP and L-PRP preparations prepared for calibration curves. $N=14$ for each type of PRP 

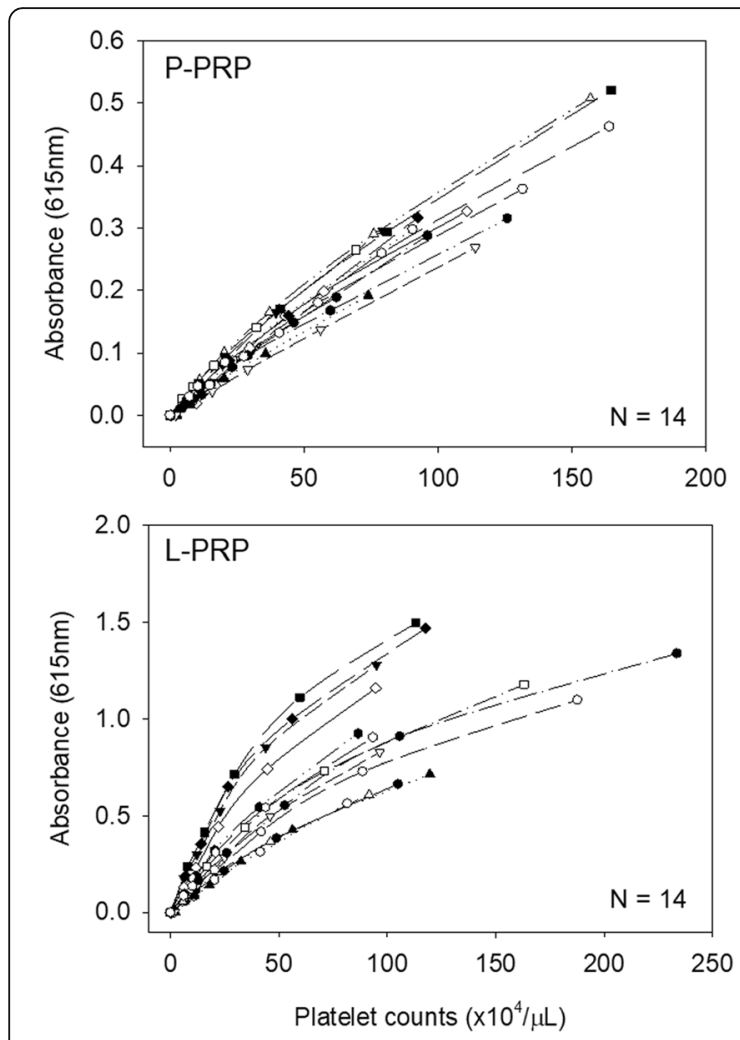

\begin{tabular}{|c|c|c|c|}
\hline$y=\mathrm{a} x+\mathrm{b}$ & $\mathrm{a}$ & $\mathrm{b}$ & $\mathrm{R}^{2}$ \\
\hline P-PRP & 0.003076 & -0.0157 & 0.9952 \\
& \pm 0.000427 & \pm 0.0146 & \pm 0.0031 \\
\hline L-PRP & 0.008521 & -0.0638 & 0.9749 \\
& \pm 0.002876 & \pm 0.0515 & \pm 0.0200 \\
\hline
\end{tabular}

Fig. 4 Calibration curves of measured platelet counts versus absorbance in P-PRP and L-PRP preparations. The samples were serially diluted by PPP, and the platelet counts were determined using an AHA and SPM. N = 14 for each type of PRP

\section{Results}

The appearance of the blood-collection tube after the first low-speed spin and representative P-PRP and L-PRP preparations after the second high-speed spin and subsequent re-suspension are shown in Fig. 2. Although low-speed spinning did not result in the formation of a clear buffy coat in the interface between the plasma and RBC fractions, the buffy coat corresponding to the plasma was not included in the second spin for P-PRP preparation. Therefore, the resulting P-PRP was light yellow in color, not reddish. In contrast, for the L-PRP preparation, the buffy coat and the surface of the $\mathrm{RBC}$ fraction just below the interface were included in the second spin. The inclusion of significant amounts of RBC turned the L-PRP red. The strength of this color was variable depending on the operators' pipetting skills; however, L-PRP preparations were more or less reddish when the maximum amount of platelets was recovered.
To characterize both the P-PRP and L-PRP preparations used for the calibration curves, blood cells were counted using an AHA (Fig. 3). For platelet counts, there was no significant difference between the two types of PRP. For WBC and RBC counts, in contrast, L-PRP contained significantly more WBCs and RBCs than P-PRP.

The samples were serially diluted, and platelets in individual dilutions were counted using the AHA. In parallel, the absorbance of each sample was measured with the SPM. The resulting calibration curves for P-PRP and L-PRP are shown in Fig. 4. Compared with P-PRP, the calibration curves for L-PRP varied with the samples and appeared generally inappropriate for linear regression. The calibration curve for P-PRP was expressed as " $y=0.00308 x-0.0157$," while that of L-PRP was " $y=$ $0.00852 x-0.638$." The SD values for both the slope and intercept values were much higher in L-PRP. In addition, the $R^{2}$ value (coefficient of determination) for the linear regression of P-PRP was 0.995 , while that of L-PRP was a little lower than that of P-PRP, 0.975, with almost 6.5-times higher SD values.

For validation of these calibration curves, P-PRP and L-PRP preparations prepared by three independent operators were employed. Blood cell counts are shown in Fig. 5. As observed in the calibration curves for the samples, significant differences were found in WBC and RBC counts, but not in platelet counts, between the P-PRP and L-PRP preparations. Correlations between platelet counts and WBC or RBC counts are shown in Fig. 6. Unexpectedly, strong positive correlations were observed only between platelet and RBC counts, but not between platelet and WBC counts, in both types of PRP preparations.

Measured versus calculated platelet counts are plotted in Fig. 7. In P-PRP preparations, the ratio of calculated platelet counts to the measured values was $108.6 \pm$ $22.0 \%$, whereas in L-PRP preparations, the ratio was $110.4 \pm 64.0 \%$. The discrepancy of SD values was reflected more clearly in the difference of $R^{2}$ values (0.671 vs. 0.0605$)$.

\section{Discussion}

Since determination of bacterial cell number is a fundamental procedure in the field of microbiology, several methods have been developed and widely employed depending on the purpose of cell counting. SPM is one of the common methods used to estimate bacterial load [12]. The advantage of SPM is speed and convenience without additional preparation steps. On the other hand, the limitations are the inability to distinguish live bacteria from dead bacteria and a relatively narrow range of detection $\left(10^{8}-10^{10}\right.$ bacteria/mL) [12]. 

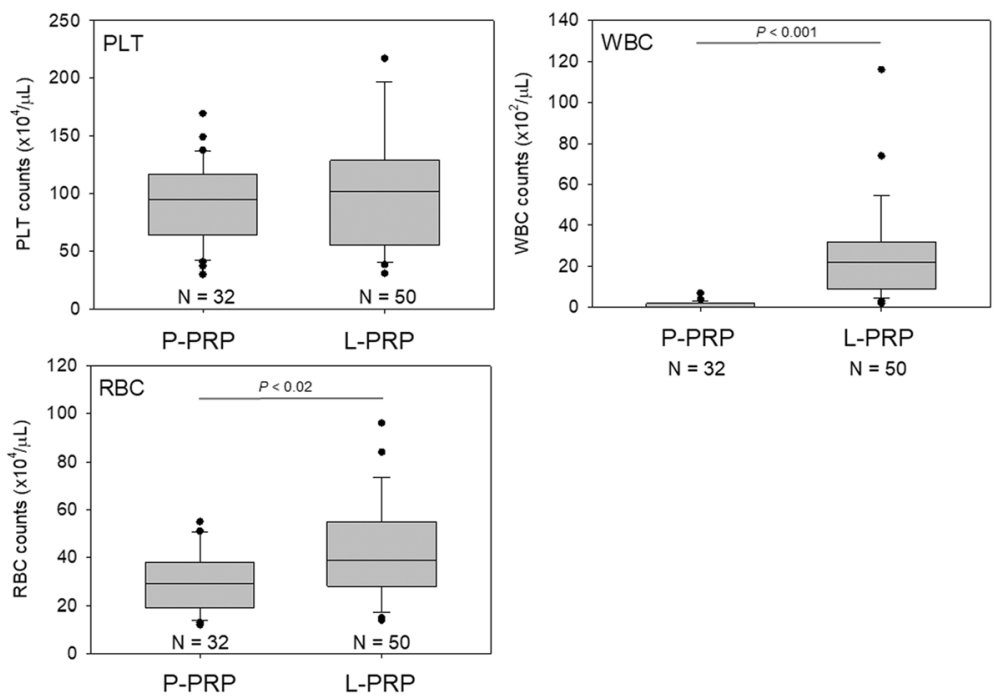

Fig. 5 Counts of platelets (PLT), WBCS, and RBCs in P-PRP and L-PRP preparations prepared for validation testing. N = 32 and 50 for P-PRP and L-PRP, respectively

A wide range of detection is not required for platelet counting in PRP preparations unlike in bacterial cell counting. However, it is more difficult to recognize platelets in PRP preparations compared to bacteria because WBCs and RBCs can more or less be included, especially when the buffy coat is included in the second spin. Lee and Tarassenko were probably inspired by the bacterial cell count and first reported the optical determination method for platelet counts [13]. However, the shortcomings of this method are that the range of RBC counts $\left(0-3 \times 10^{4} / \mu \mathrm{L}\right)$ is set below the $\mathrm{RBC}$ range $(30-$ $40 \times 10^{4} / \mu \mathrm{L}$ in average) of P-PRP and that WBCs were not taken into consideration.

To solve this problem, in this study, we separated PRP preparations into two types (i.e., P-PRP and L-PRP) for evaluation and successfully validated the spectrophotometric method in P-PRP preparations. In contrast, the accuracy of this method was lower than expected in L-PRP preparations, which is reflected in the difference in the coefficient values (Fig. 4). The striking difference
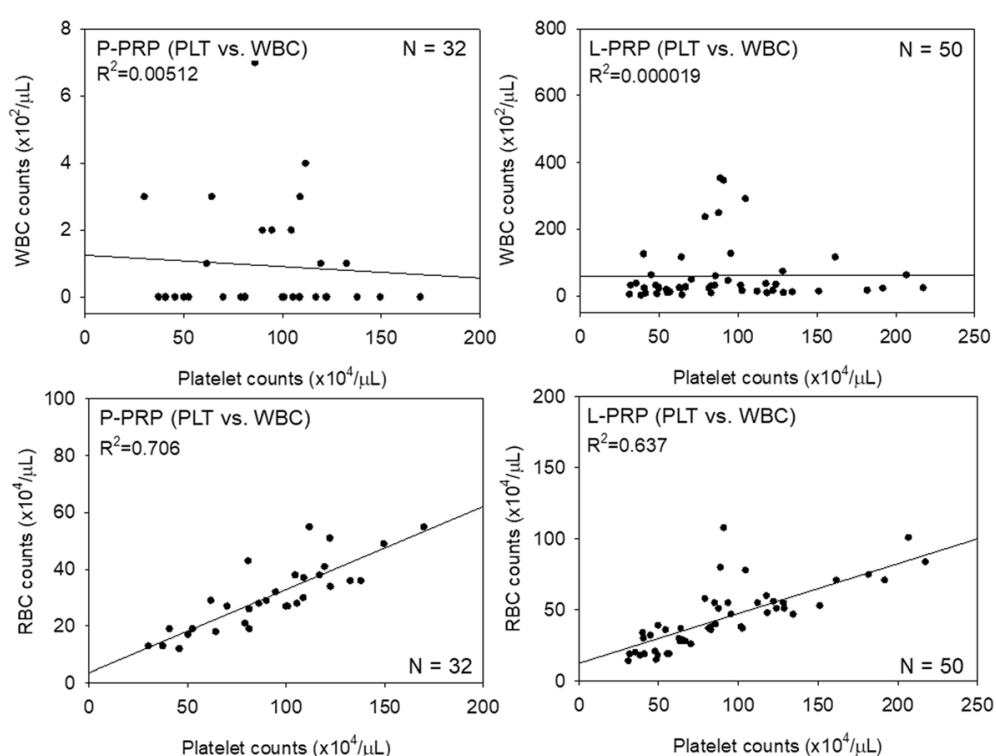

Fig. 6 Scatter plots representing possible correlations between platelet (PLT) and WBC counts and between platelet and RBC counts in P-PRP and L-PRP preparations. Note: strong positive correlations were observed between platelets and RBC in both PRP types. N = 32 and 50 for P-PRP and L-PRP, respectively 

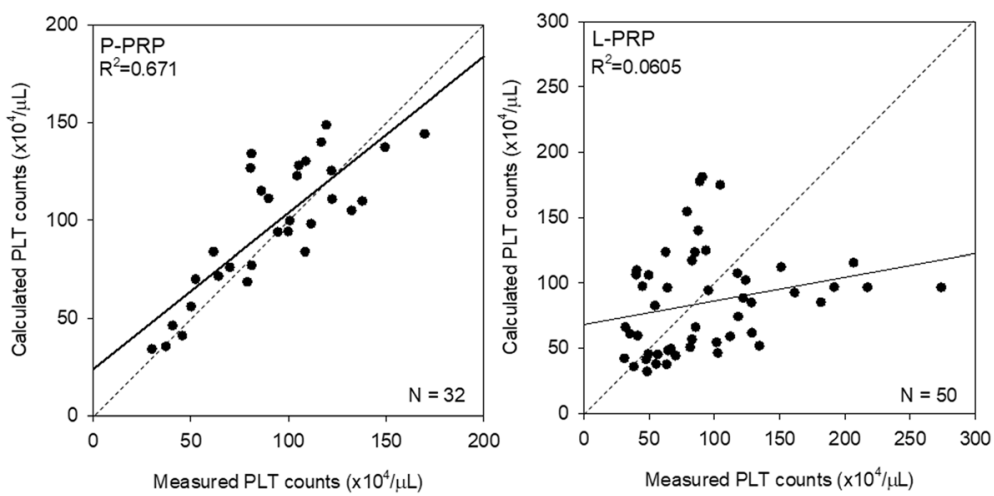

Fig. 7 Scatter plots representing correlations between measured and calculated platelet counts in P-PRP and L-PRP preparations. Note: a strong correlation was observed only in P-PRP. N $=32$ and 50 for P-PRP and L-PRP, respectively

between P-PRP and L-PRP could be attributed to the inclusion of WBCs rather than RBCs in L-PRP as RBCs were also included in P-PRP with higher platelet counts. We speculate that WBCs were the primary factor responsible for lowering the performance and that they can disrupt light transparency more effectively than can RBCs; this is because WBCs are spherical, nucleated, and larger than disk-shaped RBCs and because the absorbance of hemoglobin contained in RBCs decreases beyond $600 \mathrm{~nm}$ [17] (cf., $615 \mathrm{~nm}$, the peak wavelength used here). Besides counts, the size distribution of WBCs depends on individual donors. Hence, the ratios of large WBCs (e.g., neutrophils) to small WBCs (e.g., lymphocytes) widely vary across individuals, especially when they suffer from certain types of diseases, such as cancers, cardiovascular diseases, and pulmonary diseases [18-21].

Another limitation is the color of plasma. In terms of color, blood samples obtained from the donors participating in this study were light yellow and could be evaluated as "normal." However, we have sometimes encountered colored plasma samples in clinical practice. For example, when blood triglyceride levels are high, the plasma turns milky white or turbid [22-24]. Hemolytic plasma looks reddish, while icteric plasma appears yellow. When the degree of color change is not severe and when the transparency is maintained, the data may be compensated by the absorbance of PPP. However, in this case, we recommend the use of an AHA for accurate determination of the platelet counts.

We should discuss briefly how clinicians can perform quality assurance for individual PRP preparations. As described elsewhere [10, 25], PRP quality is evaluated mainly based on two major points: sterility and efficacy. Recent advances in PCR technology enable clinicians to quickly assess the contamination of targeted bacteria and mycoplasmas [26] in clinical settings. However, clinicians may require a well-trained operator for this kind of sterility testing. The current regulatory framework for PRP therapy in Japan requires clinicians to prepare PRP on a clean bench [10]. Therefore, as long as blood samples are handled aseptically, the resulting PRP preparations are evaluated as sterile.

As for efficacy, regardless of the assay system, several hours or days are required to complete efficacy testing. Even if it takes only several hours, unfortunately, this delay is not beneficial to many patients and is not suitable for on-site preparation and immediate use in autologous PRP therapy. The only exception is platelet counting, which takes only a few minutes with the use of an AHA. However, it is a problem that the conventional form of this device is $\$ 10,000$ or higher and requires installation space $(500 \times 500 \mathrm{~mm}$ at least). In contrast, the compact SPM used in this study costs only $\$ 800$ and can be stored in a drawer. Therefore, despite several limitations, this compact SPM would be useful for fundamental quality assurance as well as for the examination of possible correlations between platelet counts and clinical outcomes.

Consistent with the clinical significance of platelet counting, several studies have reported that the platelet concentration is the most reliable criterion for the regenerative ability of PRP $[27,28]$ because platelets increase the number of anabolic signaling molecules. Conversely, as WBCs increase the number of catabolic signaling molecules, the quality of PRP can, perhaps, be considerably altered depending on the levels of WBCs included in PRP [29]. Despite functioning to clean wounds and prevent infection, WBCs, particularly phagocytic leukocytes, have been reported to produce matrix metalloproteinases (MMPs), oxygen and nitrogen reactive species (free radicals), and proinflammatory cytokines, which could adversely affect the stem cell behavior and, consequently, tissue regeneration [27, 30, 31]. This finding is evidenced by the fact that L-PRP induces inferior effects on the bone and cartilage 
regeneration compared with P-PRP [32, 33], indicating that P-PRP is, perhaps, more suitable than L-PRP in the field of regenerative dentistry. Hence, although working only in P-PRP, our spectrophotometric method would be of great use in assuring the quality of individual PRP preparations in the dental setting.

\section{Conclusions}

In normal blood samples composed of light yellow plasma, spectrophotometric determination of platelet counts would be useful for quality assurance of individual PRP preparations. For accurate determination, however, operators should handle samples with care to minimize the inclusion of WBCs and RBCs in PRP preparations.

\section{Abbreviations}

ACD: Acid-citrate-dextrose solution; AHA: Automated hematology analyzer; LPRP: Leukocyte-rich PRP; PGE 1 : Prostaglandin $E_{1}$; PPP: Platelet-poor plasma; PRF: Platelet-rich fibrin; PRP: Platelet-rich plasma; P-PRP: Pure-PRP; RBC: Red blood cell; SD: Standard deviation; SPM: Spectrophotometer; WBC: Leukocyte

\section{Availability of data and materials}

Because an article performed and prepared in parallel is now submitted elsewhere for publication, the authors do not wish to share their data at present time.

\section{Authors' contributions}

YK, MS, TyTo, and TK conceived and designed the study, performed the experiments and data analysis, and wrote the manuscript. Kl, TaTn, TsW, $\mathrm{TkW}$, and $\mathrm{HO}$ designed and performed the experiments, data analysis, and intepretation. KN and TaTn conceived the study and participated in discussion of the results and manuscript preparation. All authors read and approved the final version of the manuscript.

\section{Ethics approval and consent to participate}

The study design and consent forms of all the procedures performed were approved by the ethics committee for human participants of the Niigata University School of Medicine (Niigata, Japan) in accordance with the Helsinki Declaration of 1964 as revised in 2013. Written informed consents to participate in the study were obtained from all the participants.

\section{Consent for publication}

Written informed consents for the publication of these were obtained from all the participants.

\section{Competing interests}

Yutaka Kitamura, Masashi Suzuki, Tsuneyuki Tsukioka, Kazushige Isobe, Tetsuhiro Tsujino, Taisuke Watanabe, Takao Watanabe, Hajime Okudera, Koh Nakata, Takaaki Tanaka, and Tomoyuki Kawase declare that they have no competing interests.

\section{Publisher's Note}

Springer Nature remains neutral with regard to jurisdictional claims in published maps and institutional affiliations.

\section{Author details}

${ }^{1}$ Tokyo Plastic Dental Society, Kita-ku, Tokyo, Japan. ${ }^{2}$ Bioscience Medical Research Center, Niigata University Medical and Dental Hospital, Niigata Japan. ${ }^{3}$ Department of Materials Science and Technology, Niigata University, Niigata, Japan. ${ }^{4}$ Division of Oral Bioengineering, Institute of Medicine and Dentistry, Niigata University, Niigata, Japan.
Received: 13 April 2018 Accepted: 29 June 2018

Published online: 02 October 2018

\section{References}

1. Marx RE, Carlson ER, Eichstaedt RM, Schimmele SR, Strauss JE, Georgeff KR. Platelet-rich plasma: Growth factor enhancement for bone grafts. Oral Surg Oral Med Oral Pathol Oral Radiol Endod. 1998:85:638-46.

2. Etulain J. Platelets in wound healing and regenerative medicine. Platelets. 2018:1-13.

3. Hou X, Yuan J, Aisaiti A, Liu Y, Zhao J. The effect of platelet-rich plasma on clinical outcomes of the surgical treatment of periodontal intrabony defects: a systematic review and meta-analysis. BMC Oral Health. 2016;16:71.

4. Pocaterra A, Caruso S, Bernardi S, Scagnoli L, Continenza MA, Gatto R. Effectiveness of platelet-rich plasma as an adjunctive material to bone graft: a systematic review and meta-analysis of randomized controlled clinical trials. Int J Oral Maxillofac Surg. 2016;45:1027-34.

5. Oryan A, Alidadi S, Moshiri A. Platelet-rich plasma for bone healing and regeneration. Expert Opin Biol Ther. 2016;16:213-32.

6. Rosello-Camps A, Monje A, Lin GH, Khoshkam V, Chavez-Gatty M, Wang HL, Gargallo-Albiol J, Hernandez-Alfaro F. Platelet-rich plasma for periodontal regeneration in the treatment of intrabony defects: a meta-analysis on prospective clinical trials. Oral Surg Oral Med Oral Pathol Oral Radiol. 2015, 120:562-74

7. Jovani-Sancho MD, Sheth CC, Marques-Mateo M, Puche-Torres M. Plateletrich plasma: a study of the variables that may influence its effect on bone regeneration. Clin Implant Dent Relat Res. 2016;18:1051-64.

8. De Pascale MR, Sommese L, Casamassimi A, Napoli C. Platelet derivatives in regenerative medicine: an update. Transfus Med Rev. 2015;29:52-61.

9. Del Fabbro M, Corbella S, Taschieri S, Francetti L, Weinstein R. Autologous platelet concentrate for post-extraction socket healing: a systematic review. Eur J Oral Implantol. 2014:7:333-44.

10. Kawase T, Okuda K. Comprehensive quality control of the regenerative therapy using platelet concentrates: the current situation and prospects in Japan. Biomed Res Int. 2018; in press

11. Kitamura Y, Watanabe T, Nakamura M, Isobe K, Kawabata H, Uematsu K Okuda K, Nakata K, Tanaka T, Kawase T. Platelet counts in insoluble plateletrich fibrin clots: a direct method for accurate determination. Front Bioeng Biotechnol. 2018;6:4

12. Hazan R, Que YA, Maura D, Rahme LG. A method for high throughput determination of viable bacteria cell counts in $96-$ well plates. BMC Microbiol. 2012;12:259.

13. Lee VS, Tarassenko L. An optical method for the determination of platelet count in platelet samples contaminated with red blood cells. J Biochem Biophys Methods. 1992:24:215-23.

14. Davis VL, Abukabda AB, Radio NM, Witt-Enderby PA, Clafshenkel WP, Cairone JV, Rutkowski JL. Platelet-rich preparations to improve healing. Part I: workable options for every size practice. J Oral Implantol. 2014;40:500-10.

15. Kossev P, Sokolov T. Platelet-rich plasma (PRP) in orthopedics and traumatology_review. In: Metodiev K, editor. Immunopathology and immunomodulation: IntechOpen limited; 2015. p. 173-95.

16. Parrish WR, Roides B, Hwang J, Mafilios M, Story B, Bhattacharyya S. Normal platelet function in platelet concentrates requires non-platelet cells: a comparative in vitro evaluation of leucocyte-rich (type 1a) and leucocytepoor (type 3b) platelet concentrates. BMJ Open Sport Exerc Med. 2016;2: e000071.

17. Zijlstra WG, Buursma A. Spectrophotometry of hemoglobin: absorption spectra of bovine oxyhemoglobin, deoxyhemoglobin, carboxyhemoglobin, and methemoglobin. Comp Biochem Physiol B: Biochem Mol Biol. 1997;1 18:743-9.

18. Afari ME, Bhat T. Neutrophil to lymphocyte ratio (NLR) and cardiovascular diseases: an update. Expert Rev Cardiovasc Ther. 2016;14:573-7.

19. Faria SS, Fernandes PC, Silva MJB, Lima VC, Fontes W, Freitas-Junior R, Eterovic AK, Forget $P$. The neutrophil-to-lymphocyte ratio: a narrative review. Ecancermedicalscience. 2016;10:702

20. Gao Y, Wang W-J, Zhi Q, Shen M, Jiang M, Bian X, Gong F-R, Zhou C, Lian L, Wu M-Y, Feng J, Tao M, Li W. Neutrophil/lymphocyte ratio is a more sensitive systemic inflammatory response biomarker than platelet/ lymphocyte ratio in the prognosis evaluation of unresectable pancreatic cancer. Oncotarget. 2017:8:88835-44.

21. Paliogiannis $P$, Fois AG, Sotgia $S$, Mangoni AA, Zinellu E, Pirina P, Negri S, Carru C, Zinellu A. Neutrophil to lymphocyte ratio and clinical outcomes in COPD: recent evidence and future perspectives. Eur Respir Rev. 2018;27 
22. Vassallo RR, Stearns FM. Lipemic plasma: a renaissance. Transfusion. 2011;51: 1136-9.

23. Guder WG, da Fonseca-Wollheim F, Heil W, Schmitt YM, Töpfer G, Wisser H, Zawta B. The Haemolytic, Icteric and Lipemic Sample Recommendations Regarding their Recognition and Prevention of Clinically Relevant Interferences. Recommendations of the Working Group on Preanalytical Variables of the German Society for Clinical Chemistry and the German Society for Laboratory Medicine. LaboratoriumsMedizin / Journal of Laboratory Medicine. 2009;24:357-64.

24. Simundic AM, Nikolac N, Ivankovic V, Ferenec-Ruzic D, Magdic B, Kvaternik M, Topic E. Comparison of visual vs. automated detection of lipemic, icteric and hemolyzed specimens: can we rely on a human eye? Clin Chem Lab Med. 2009;47:1361-5.

25. Kawase T, Watanabe T, Okuda K. Platelet-rich plasma and its derived platelet concentrates: what dentists involved in cell-based regenerative therapy should know. Nihon Shishubyou Gakkai Kaishi. 2017;59:68-76. (in Japanese)

26. Tokuno O, Hayakawa A, Yanai T, Mori T, Ohnuma K, Tani A, Minami H, Sugimoto T. Sterility testing of stem cell products by broad-range bacterial 16S ribosomal DNA polymerase chain reaction. Lab Med. 2015;46:34-41.

27. Davis VL, Abukabda AB, Radio NM, Witt-Enderby PA, Clafshenkel WP, Cairone JV, Rutkowski JL. Platelet-rich preparations to improve healing. Part II: platelet activation and enrichment, leukocyte inclusion, and other selection criteria. J Oral Implantol. 2014;40:511-21.

28. Sundman EA, Cole BJ, Fortier LA. Growth factor and catabolic cytokine concentrations are influenced by the cellular composition of platelet-rich plasma. Am J Sports Med. 2011;39:2135-40.

29. Kobayashi Y, Saita Y, Nishio H, Ikeda H, Takazawa Y, Nagao M, Takaku T, Komatsu N, Kaneko K. Leukocyte concentration and composition in plateletrich plasma (PRP) influences the growth factor and protease concentrations. J Orthop Sci. 2016;21:683-9.

30. Anitua E, Zalduendo MM, Prado R, Alkhraisat MH, Orive G. Morphogen and proinflammatory cytokine release kinetics from PRGF-Endoret fibrin scaffolds: evaluation of the effect of leukocyte inclusion. J Biomed Mater Res A. 2015;103:1011-20.

31. Kizil C, Kyritsis N, Brand M. Effects of inflammation on stem cells: together they strive? EMBO Rep. 2015;16:416-26.

32. Xu Z, Yin W, Zhang Y, Qi X, Chen Y, Xie X, Zhang C. Comparative evaluation of leukocyte- and platelet-rich plasma and pure platelet-rich plasma for cartilage regeneration. Sci Rep. 2017;7:43301.

33. Yin W, Qi X, Zhang Y, Sheng J, Xu Z, Tao S, Xie X, Li X, Zhang C. Advantages of pure platelet-rich plasma compared with leukocyte- and platelet-rich plasma in promoting repair of bone defects. J Transl Med. 2016;14:73.

\section{Submit your manuscript to a SpringerOpen ${ }^{\circ}$ journal and benefit from:}

- Convenient online submission

- Rigorous peer review

- Open access: articles freely available online

- High visibility within the field

- Retaining the copyright to your article

Submit your next manuscript at $\gg$ springeropen.com 